\title{
Disegno di fortificazioni nella cartografia tra i secoli XII e XVI
}

Drawing of fortifications in the cartography between the twelfth and sixteenth centuries

\author{
Arturo Gallozzi ${ }^{\text {a }}$, Michela Cigola ${ }^{b}$ \\ Università degli Studi di Cassino e del Lazio Meridionale, Cassino, Italy \\ a gallozzi@unicas.it; ${ }^{\mathrm{b}}$ cigola@unicas.it
}

\begin{abstract}
The contribution schematically analyzes themes and graphic codes related to the cartographic representation, in a period between twelfth and sixteenth centuries. Significant examples of Maps, Nautical charts, Atlases and Globes are examined, highlighting some characteristic aspects, connected to the figuration of fortified and non-fortified cities. In cartography the period considered is extremely significant. Because there is the passage from a predominantly symbolic representation of the world to a "modern" evolution of maps, based on direct observations using measuring instruments.
\end{abstract}

Keywords: Mappae mundi, itineraria picta, nautical maps, cartography, Jerusalem, city icons.

\section{Introduzione}

Il contributo ripercorre sinteticamente, tra i secoli XII e XVI, l'evoluzione della rappresentazione cartografica. Prendendo spunto da vari e significativi esempi di Mappe, Carte nautiche, Atlanti e Mappamondi, si cerca di evidenziare alcuni aspetti salienti, caratterizzanti temi e codici grafici, connessi anche alla figurazione di città fortificate e non.

Per quanto attiene alla rappresentazione delle mappae mundi possono essere individuati due principali criteri di classificazione: uno in prevalenza grafico, l'altro più propriamente temporale. La prima suddivisione si articola in mappae tripartite o T-O (schematiche e non schematiche); mappae a zone; mappae quadripartite; mappae di transizione. Mentre nella ripartizione cronologica si distinguono: il periodo tardo grecoromano ed il periodo patristico (400-700 ca.); il Rinascimento carolingio (700 ca.-1096); l'Età delle crociate (1096-1270); e il periodo di transizione dal Medioevo al Rinascimento (13001460). La storia e l'evoluzione della cartografia nei secoli è pertanto variamente articolata e presenta molteplici livelli di lettura (mondo religioso, regno animale, terre incognite, aspetti illustrativi, militari, commerciali etc.). Nel contributo si evidenziano solo alcuni esempi paradigmatici dei periodi più significativi che hanno segnato un punto di riferimento e di svolta nella rappresentazione cartografica, con particolare attenzione agli aspetti e agli eventi legati al Mediterraneo e alla figurazione delle principali città. In cartografia, il periodo che va dal 1300 al 1500 è estremamente significativo. Si passa, infatti, dalle carte medievali, il cui fine era mostrare attraverso un'illustrazione o più semplicemente decorare il testo dei codici, alle carte cosiddette "moderne" basate su osservazioni dirette e redatte con l'ausilio di strumenti di misurazione. Molteplici sono i fattori di interesse che attraversano i secoli di studio: il mondo medievale si mette in marcia; si organizzano viaggi commerciali e diplomatici; si stabiliscono contatti con paesi extraeuropei; i viaggiatori cominciano a riportare 
informazioni che trasformeranno, nel XV secolo, le carte medievali e permetteranno di migliorarle, sostituendo via via i dati e i codici di rappresentazione dell'antichità. Proprio in questo lasso di tempo si verificano, inoltre, due eventi che avranno una peso straordinario sulla cartografia e sulla storia dell'umanità: nel 1455 ca. Johannes Gutenberg (1400 ca.-1468) inventa, a Mainz, la stampa a tipi mobili, già in uso in Cina fin dall'XI secolo; nel 1492 Cristoforo Colombo (1451-1506) scopre il continente americano, segnando lo spostamento dell'asse economico europeo e l'apertura di nuovi orizzonti cartografici.

\section{Tipologie di carte}

\subsection{Mappe T-O}

Nelle carte medievali antecedenti il 1300 le terre si riconoscono a fatica, e non si ritrova che qualche dettaglio esatto. La maggior parte di queste mappe è una cornice in cui trovano posto credenze e miti popolari. La tipologia più diffusa in questo periodo è chiamata in vari modi: carte ecumeniche, mappae mundi, o mappe T-O. Queste carte, spesso estremamente schematiche hanno l'Est in alto, consistono in un cerchio che rappresenta il fiume Oceano che racchiude il mondo conosciuto, tripartito secondo la suddivisione della Terra fra i tre figli di Noè: Sem-Asia, Cham-Africa, Japhet-Europa (Fig. 1). Il Mediterraneo, che divide l'Europa dall'Africa, costituisce il braccio verticale dalla $\mathrm{T}$, mentre nel braccio orizzontale è raffigurato il mare magnum, in altre rappresentazioni si trovano i fiumi Tanais (l'attuale Don) e Nilo, che limitano inferiormente l'Asia. Questo tipo di mappe avrà una diffusa proliferazione per tutto il medioevo, in codici e miniature. Un primo esempio a stampa è pubblicato, come una piccola illustrazione marginale, all'inizio del XIV libro de terra et partibus nelle Etymologiae di Isidoro di Siviglia (560-636 d.C.). L'opera, scritta nell'VII secolo, fu stampata circa 800 anni dopo ad Augsburg nel 1472 (Fig. 2).

La schematica rappresentazione delle prime mappe tripartite, viene progressivamente superata con l'aggiunta di città e dettagli simbolici e orografici. Appaiono decorazioni, creature mito- logiche, mentre le città più importanti vengono rappresentate con torri e fortificazioni.

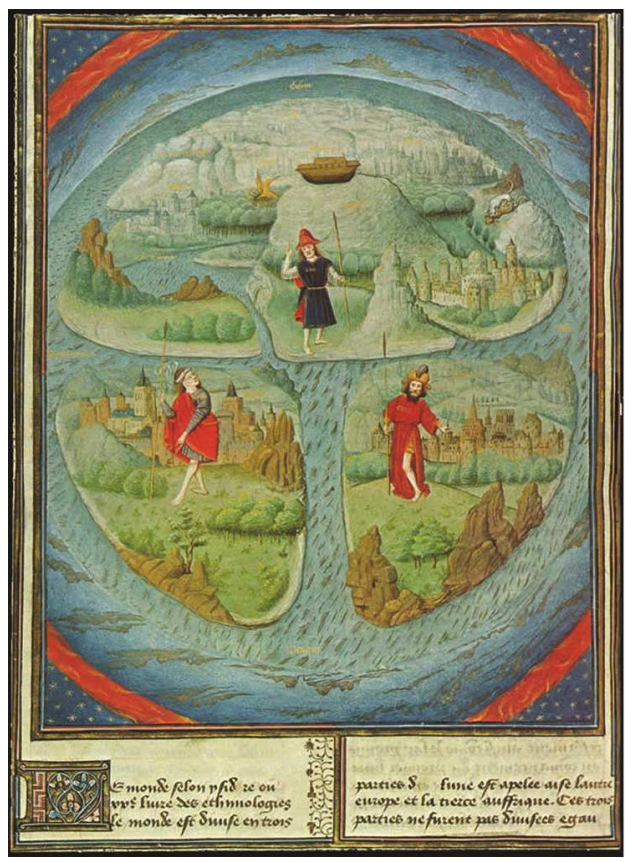

Fig. 1. La Terra divisa tra i figli di Noè, Mappa mundi in Jean Mansel, La Fleur des Histoires, 1459-1463 (Bibliothèque Royale, Belgique, ms. 9231, fol. 281v).

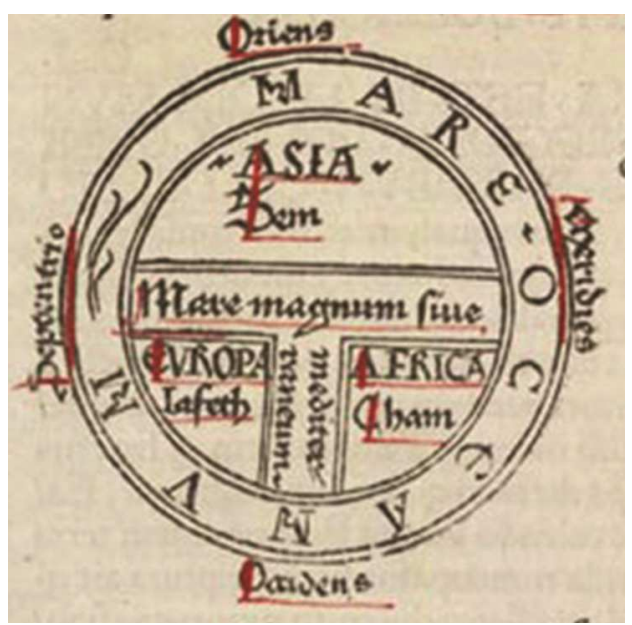

Fig. 2. Mappa mundi, Etymologiae, lib. XIV, 1472.

Tra la copiosa e variegata categoria delle mappae mundi, caratterizzate dalla configurazione triparti- 
ta con immagini di città, predomina la rappresentazione della città di Gerusalemme.

Nella figura 3 è il mappamondo di Saint Denis (metà del XIII secolo). La mappa illustra l'ultimo folio delle Chroniques de St-Denis, manoscritto appartenente a Carlo V il Saggio (1338-1380) e conservato nella biblioteca Sainte-Geneviève di Parigi. La presenza di rappresentazioni cartografiche e mappae mundi nelle cronache medievali era ricorrente, poiché, come già aveva insegnato Cicerone, "la natura delle cose richiede l'ordine del tempo e la descrizione dei paesi". Anche questa rappresentazione presenta il mondo tripartito e contornato dal fiume Oceano oltre il quale, entro cartigli semicircolari, sono i nomi dei dodici venti principali. La nomenclatura in caratteri gotici indica i paesi con i soli nomi senza distinzioni tra nazioni e continenti.

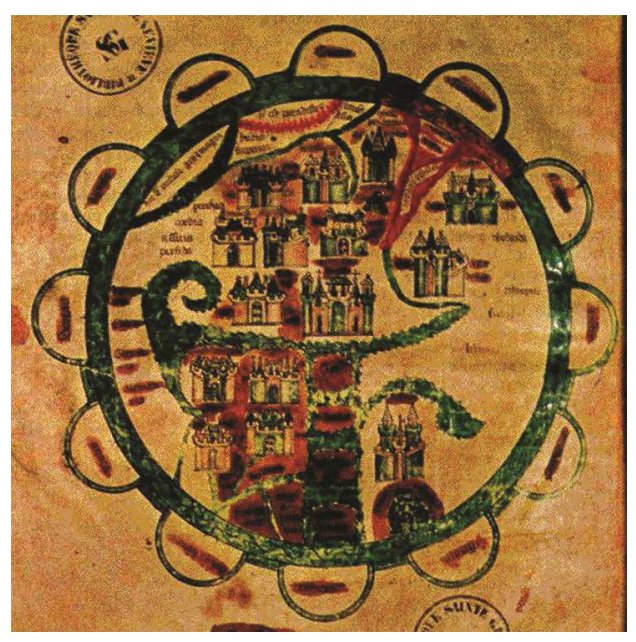

Fig. 3. Mappamondo di Saint-Denis 1275-1280 (ms.782, f. 374v, Biblioteca Sainte-Geneviève, Paris).

Vi sono quindici viste figurate di città, tutte ugualmente turrite e cinte di mura, una diversa dall'altra. Sono rappresentate 10 città in Asia, 4 in Europa ed una in Africa, Gerusalemme occupa la posizione geocentrica. Tuttavia l'illustrazione, come altre simili, non riveste il ruolo di descrivere lo stato delle conoscenze geografiche alla fine del XIII secolo o all'inizio del XIV secolo, ma delinea altre finalità: religiose, celebrative etc. E' anche in quest'ottica che la citta di Gerusalemme dall'XI secolo, sin dalle prime Crociate, è posta al centro dell'universo. Nel cuore geometrico, come "l'ombelico della terra", nel punto del cerchio perfetto che rappresenta la sfera terrestre.

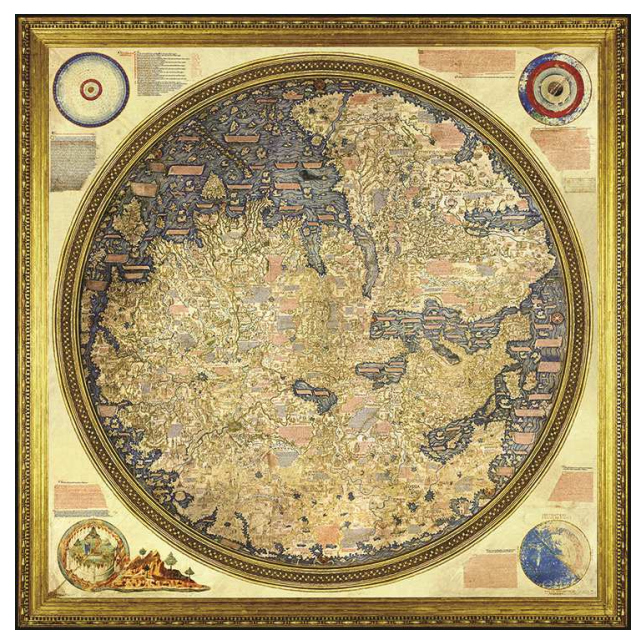

Fig. 4. Mappa mundi di Fra Mauro, 1450 ca. (Biblioteca Nazionale Marciana, Venezia).

La transizione dai vecchi mappamondi alle nuove rappresentazioni cartografiche è testimoniata da una delle opere più belle e importanti della storia della cartografia, la Mappa mundi di Fra Mauro (1400-1464 ca.), ibrido fra una mappa mundi complessa e un portolano (Fig. 4). La mappa, realizzata nel monastero veneziano di San Michele all'isola presso Murano nel 1450 ca., delinea un'immagine completa del mondo secondo quanto si credeva conoscere nel tardo Medioevo cristiano. L'orientamento vede il sud in alto, mentre la città di Gerusalemme non è più posta al centro del mondo, contrariamente alle molteplici altre mappae mundi medievali. La città santa resta un centro ideale, ma è il mare Mediterraneo a divenire il fulcro della rappresentazione terracquea, con un netto spostamento verso occidente. Basata sui resoconti attendibili di molti viaggiatori in Africa e in Asia, la mappa riporta minuziose descrizioni dei due continenti e forse la prima immagine del Giappone (Cimpagu) disegnata da un cartografo occidentale.

\subsection{Portolani e Carte nautiche}

Tra la fine del Medioevo e l'inizio del Rinascimento, le mappae mundi caddero progressiva- 
mente in disuso, attraverso un graduale processo di trasformazione, soppiantate da rappresentazioni più evolute. A poco a poco scompaiono tutti quegli elementi paradigmatici, legati alla tradizione classica e agli aspetti religiosi, che ne avevano determinato la ripetuta riproposizione. Nondimeno prosegue per lungo tempo la coesistenza tra i due tipi principali di prodotti cartografici (la carta immagine-mappae mondi, opera di eruditi, e la carta strumento, opera di cartografi professionisti). Tuttavia le nuove mappe sostituirono gradualmente l'est con il nord come elemento fondamentale di orientamento. Ciò derivò sia dalle prescrizioni di Tolomeo (100 ca.178 d.C.), che vedeva le carte orientate con il nord in alto, ma soprattutto dalla diffusione della bussola nei sistemi di navigazione, che privilegiava il nord come direzione principale. $\mathrm{Si}$ affermano, così, nel tardo medioevo, nuove categorie documentali: i portolani e le carte nautiche. Mentre i portolani costituivano prevalentemente descrizioni in forma discorsiva di rotte caratterizzate dalla navigazione da porto a porto, le carte nautiche rappresentano generalmente la sintesi grafica di quanto descritto nei portolani. Quest'ultime, dotate di una forte valenza innovativa, pur se fondate su semplici elementi figurativi, aprirono la strada alla cartografia moderna, in forte discontinuità con la produzione cartografica antecedente, introducendo caratteristiche informative basate sulla corrispondenza spaziale della realtà geografica. La loro principale peculiarità è la presenza di segni caratteristici: una precisa delineazione del profilo costiero, la dettagliata toponomastica litoranea, l'intreccio di linee, originate da una o più rose dei venti, secondo la direzione dei principali venti del Mediterraneo. Strumenti per i naviganti, concentrano l'interesse sui dettagli delle coste, le più antiche sono di un'estrema semplicità, quasi del tutto prive di elementi superflui, solo col tempo vennero introdotti vari apparati decorativi. In generale è forte il contrasto fra la descrizione del fronte costiero, affollato di informazioni e particolari, rispetto all'entroterra spesso privo di ogni dettaglio.

La "Carta del Mediterraneo orientale" (Fig. 5) è la più antica carta nautica di data certa, firmata e datata al 1311 da Pietro Vesconte (XIV secolo), cartografo e geografo genovese, che con la sua opera influenzò la cartografia nautica italiana e catalana nel corso dei secoli XIV e XV.

Tra le carte nautiche più tarde, degna di nota è la "Mappa dell'Oceano Atlantico nord-orientale, del Mar Mediterraneo e del Mar Nero" di Salvatore de Pilestrina del 1511 (Fig. 6).

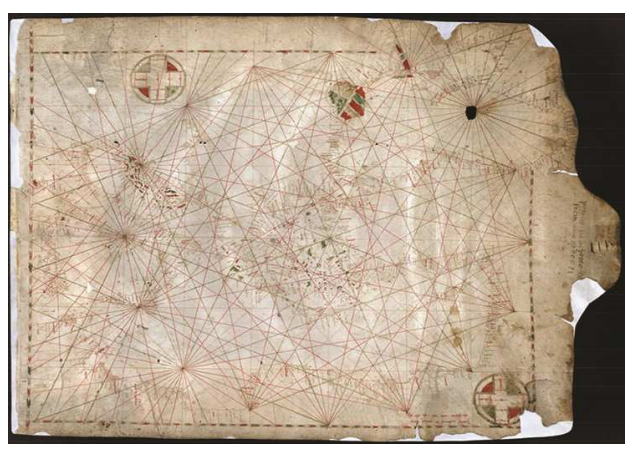

Fig. 5. Pietro Vesconte, Carta nautica del Mediterraneo orientale, del mar Nero e del mar d'Azov, 1311 (Archivio di Stato di Firenze).

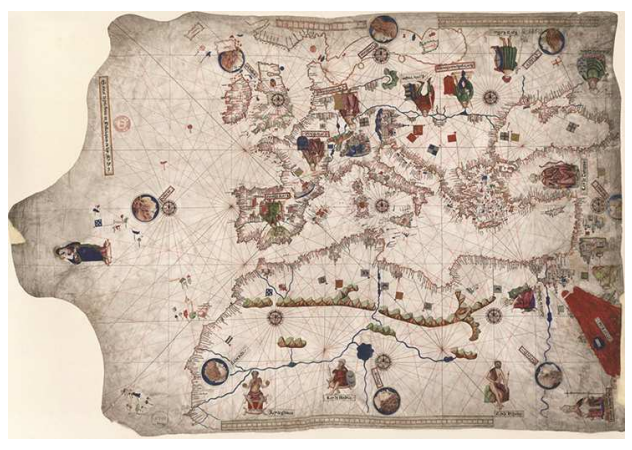

Fig. 6. Salvatore de Pilistrina, Carta nautica del mare Mediterraneo e mar Nero, 1511, fac-simile di Otto Progel del 1843 (CPL GE AA-563 RES, Bibliothèque Nationale de France).

La cartografia nautica di questo periodo ha raggiunto un vocabolario iconografico maturo (apparato decorativo, rose dei venti, etc.), che trova stilemi comuni tra le varie produzioni cartografiche, che differiscono essenzialmente per le capacità artistiche degli autori.

\subsection{Itineraria picta}

Un altro corpus cartografico importante è riferibile alle carte itinerario. Queste rappresentano 
una delle forme più antiche di descrizione geografica, con essenziali riferimenti a strade, città, luoghi, manufatti etc. lungo un dato percorso. Redatte sia per uso privato che per finalità militari o amministrative, o ancora per fini religiosi di pellegrinaggio o di conquista. Sono da distinguersi due diversi tipi di itinerari: scritti (itineraria adnotata) e figurati (itineraria picta) cioè con rappresentazione cartografica. Tra questi ultimi, forse il più famoso è la Tabula Peutingeriana, scoperta nel 1507, conservata attraverso una copia del XIII secolo, restituisce l'immagine di uno spazio geografico risalente all'epoca tardo-romana, IV-V secoli d.C. (Fig. 7).

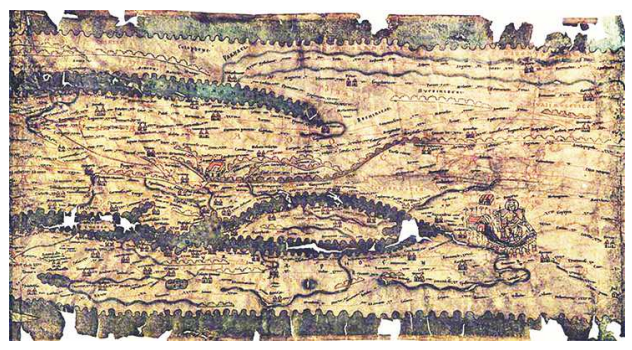

Fig. 7. Tabula Peutingeriana. Segmento IX, con Gerusalemme (Aelia Capitolina) e Antiochia.

Composta da 11 segmenti in pergamena, mostra tutte le strade dell'impero romano, ma anche città, mari, fiumi e catene montuose. Le località sono riconoscibili attraverso un preciso codice grafico delle "vignette", in relazione all'importanza e alla funzione di quanto rappresentato. Le strade e i fiumi si identificano rispettivamente con una linea rossa e una striscia blu; altre raffigurazioni simboliche sono distinte in macrofamiglie: are e altari, doppie torri, aquae (terme), edifici a tempio, porti e fari, horrea (magazzini), città rappresentate con cinte di mura turrite e alcune personificazioni. Le icone presentano rappresentazioni planimetriche, proiezioni frontali, pseudo-assonometrie, nel tentativo di rendere la tridimensionalità del rappresentato, tutto in funzione progressiva in relazione all'importanza del riferimento. Nella Tabula la presenza di Gerusalemme è di scarsa importan$\mathrm{za}$, il testo accanto alla vignetta riporta "Prima si chiamava Gerusalemme, ma ora Aelia Capitolina", fa riferimento all'imperatore Adriano che diede questo nome alla città. Diventa compren- sibile che nel periodo della redazione della mappa, il cristianesimo, anche se presente, non si trovava nel centro di interesse, nel contesto dell'impero romano.

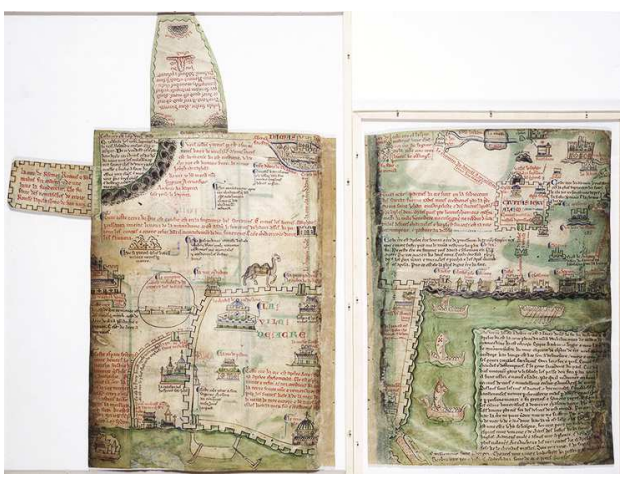

Fig. 8. Mattew Paris, Iter de Londinio in Terram Sanctam. A sx il porto di Acri (Israele), a dx al centro, la città di Gerusalemme, XIII secolo (Londra, BL, ms. Royal 14 C. VII, ff 4 v e $5 r$ ).

In epoca medievale, sul piano cartografico, mentre le carte nautiche aprivano nuovi orizzonti alla rappresentazione geografica, anche nella cartografia terrestre si assiste a un recupero e reinterpretazione delle antiche tradizioni degli itineraria picta. Un contesto particolare è rappresentato, nell'itinerario figurato di Matthew Paris, monaco benedettino (1200 ca.-1259), "Iter de Londinio in Terram Sanctam", che percorre i luoghi da Londra a Gerusalemme. Il cammino si sviluppa tra le principali città europee e i maggiori centri di pellegrinaggio della cristianità romana, in un viaggio che attraversa anche i margini del Mediterraneo (Fig. 8). Gerusalemme illustra la fine del viaggio, circondata da un muro merlato etichettato Civitas Ierusalem, mentre le altre città, costiere e non, sembrano essere rappresentate secondo un codice gerarchico, con spesso una compresenza di torri merlate e campanili, volendo forse distinguere gli edifici civili da quelli religiosi.

\subsection{L'opera di Tolomeo}

Nel 1453 gli Ottomani e un gran numero di manoscritti bizantini arrivarono in Italia, fra questi anche la "Geografia" di Tolomeo, che giunse a Firenze agli inizi del XV secolo. Del trattato di 
Tolomeo esistevano due versioni: la "A" con 27 mappe e la "B" con 64; solo la prima arrivò in Europa (Fig. 9).

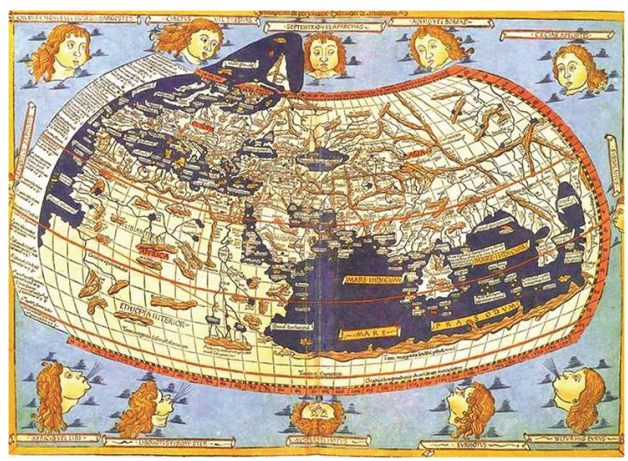

Fig. 9. Tolomeo, Cosmographia, Johannes Schnitzer, Ecumene secondo la descrizione di Tolomeo, 1482.

Il testo di Tolomeo, considerato il canone della cartografia e l'opera fondamentale della geografia, fu riprodotto senza varianti per più di un secolo e mezzo. Le carte ebbero un destino differente, pur ritenute documenti della cartografia greco-romana, sin dalla metà del XV secolo furono accompagnate da altre carte dette moderne, che avevano una nomenclatura coeva e correggevano i dettagli che non corrispondevano più alla realtà conosciuta dell'epoca. La più importante conseguenza dell'introduzione dell'opera di Tolomeo in occidente fu lo sviluppo di un metodo per la proiezione della sfera terrestre su un piano, ricorrendo ai principi fondamentali della geometria e della matematica. Da allora le mappe furono costruite su un reticolo di base usando meridiani e paralleli in termini di latitudine e longitudine. Si trattò di una novità importante per il mondo della cartografia medievale: disegnare una griglia geometrica complessa entro la quale posizionare gli elementi geografici: città, fiumi, monti, regioni. Inoltre, queste nuove regole di disegno basandosi sull'uso di coordinate geografiche richiedevano il posizionamento degli elementi rappresentati, non più riferendosi agli itinerari o alle descrizioni di fonti letterarie, ma in rapporto ad una precisa mutua collocazione.

\subsection{Atlanti e Mappamondi}

Le mappe cominciano ad essere documenti e fonte di informazioni che si estendono oltre il bacino del Mediterraneo, fino ad arrivare in Scandinavia e talvolta fino alla Cina. Nella produzione delle molteplici carte, che testimoniano questo periodo di nuova e più articolata rappresentazione del mondo conosciuto, un'influenza significativa riveste la scuola cartografica catalana, con il suo più illustre esponente: il cartografo ebreo Abrahm Cresques (1325-1387). A lui e suo figlio Jahuda si deve uno tra i più significativi esempi della cartografia medievale, conosciuto come Atlante Catalano (Fig. 10), redatto nel 1375 ca., che costituirà la base di una lunga serie di mappe che ad esso farà riferimento. Nell'Atlante la rappresentazione delle città segue essenzialmente un codice pressoché univoco (una cinta merlata con all'interno due torri e un campanile centrale), differenziando soltanto le dimensioni in funzione dell'importanza, mentre la città di Gerusalemme è raffigurata da un semplice tempio.

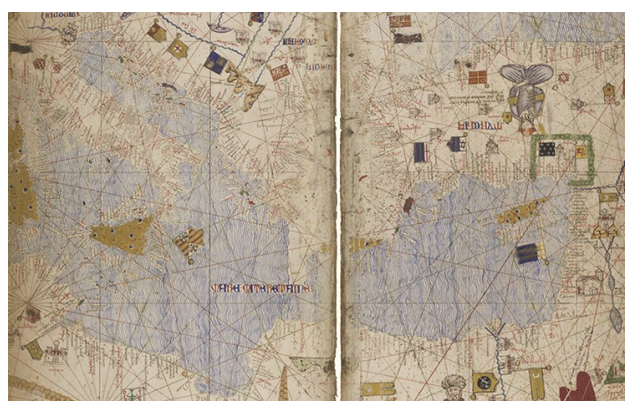

Fig. 10. Abrahm Cresques, Atlante Catalano, 1375, particolare F. 4v-5 (Bibliothèque Nationale de France, Département des Manuscrits. Espagnol 30).

\subsection{La cartografia delle grandi scoperte}

Tra la fine del XIV secolo e la fine del XVI avvennero notevoli cambiamenti, identificando spesso tale periodo come "età delle scoperte geografiche". E' proprio dopo le scoperte del Nuovo Mondo, che nel 1494 venne sottoscritto il trattato di Tordesillas. L'Impero spagnolo e l'Impero portoghese, in una feroce lotta coloniale che coinvolse la politica, la religione, la morale e soprattutto l'economia, si divisero il mondo -al di fuori dell'Europa- in un duopolio esclusivo. In questo nuovo contesto geo-politico, più di prima, le conoscenze geografiche assunsero notevole importanza, tanto da sviluppare fenomeni 
di spionaggio cartografico. Testimonianza di ciò sono le vicende legate al Planisfero di Cantino (Carta de navigare per le isole Novamente trovate in la parte de l'India) (Fig. 11).

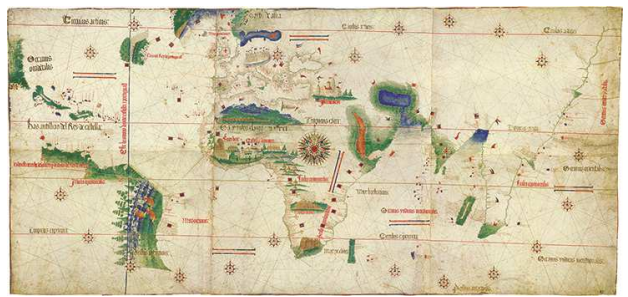

Fig. 11. Carta del Cantino (Bib. Estense Universitaria, Modena - C.G.A.2 Ms., 1502-1505 ca.).

Alberto Cantino (XV-XVI secolo), trafugò a Lisbona nel 1502 su mandato di Ercole d'Este, duca di Ferrara (1431-1505), le informazioni geografiche per descrivere la forma del mondo agli inizi del XVI secolo. Nella mappa che fece redigere da un cartografo portoghese, con una linea verticale blu di spartizione da nord a sud, veniva riprodotta anche la situazione determinata dal trattato di Tordesillas.

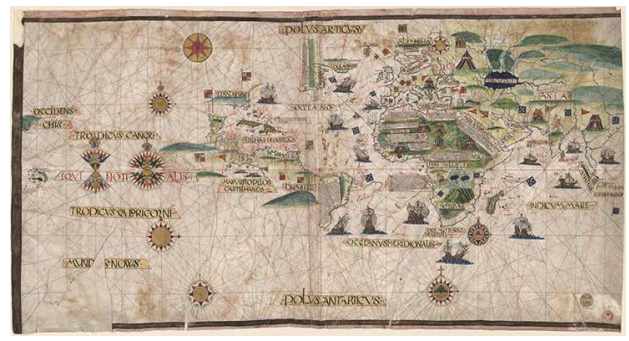

Fig. 12. Jorge Reinel, "Carta del Mondo", 1519, facsimile di Otto Progel (CPL GE AA-564 RES, Bibliothèque Nationale de France).

Nel 1519 il cartografo portoghese Jorge Reinel (1502-1572) produsse un'importante "Carta del Mondo" (Fig. 12), forse anche con l'ausilio del suo più famoso padre Pedro (1462-1442 ca.).

La produzione di Reinel risulta di particolare interesse, in quanto non solo partecipò ai preparativi e alla redazione delle mappe per i viaggi del suo connazionale Ferdinando Magellano (14801521), ma anche perché le sue carte furono usate per sostenere le rivendicazioni della corona di
Castiglia in merito alle Isole Molucche in seguito al trattato di Tordesillas.

\section{Le "vignette"}

Dall'esame delle carte, considerate in questo breve e non esaustivo escursus storicocartografico, è stata tralasciata l'analisi delle vignette delle città fortificate nell'ambito del bacino Mediterraneo che, rivestendo caratteristiche e spunti di analisi degni di nota, richiederebbe una descrizione analitica più articolata. Pertanto, a titolo esemplificativo si è scelto di evidenziare alcuni aspetti grafici relativi ad una città particolarmente significativa nel panorama storico esaminato. La città di Gerusalemme, all'interno delle coste del Mediterraneo orientale, ha rappresentato infatti un fulcro emblematico che ha caratterizzato molteplici apparati cartografici, in particolare nell'antichità, con la prima fase delle rappresentazioni cristiane $\mathrm{O}-\mathrm{T}$, e poi nelle mappe del periodo delle crociate. Nello schema di figura 13 è sintetizzato un parziale repertorio di varie rappresentazioni della città di Gerusalemme (alcune certe, altre ipotizzate). Le varie immagini, non consentono di delineare un'evoluzione codificata delle rispettive icone, sia in termini temporali, che in funzione di una rappresentazione aderente alla realtà. Tuttavia, nella generalità delle carte esaminate, prevalgono le figurazioni fortificate, mentre altre vignette seguono un simbolismo coerente con i codici grafici della carta a cui appartengono, altre ancora si discostano da ogni riferimento canonico, assumendo propria singolarità grafica in ragione della specificità del sito.

\section{Conclusioni}

Lo sviluppo e l'affinamento della tecnica cartografica quasi mai è disgiunto da scopi religiosi, militari, economici o dimostrativi. La rappresentazione delle città, dei presidi e degli elementi fortificati, permea e percorre tutto il periodo preso in esame, svolgendo un ruolo da protagonista, sia nell'epoca medievale che negli anni successivi alle grandi scoperte. L'apparato e il corredo iconografico di queste carte esalta, quindi, graficamente la rappresentazione dei territori descritti, con vignette e icone, di forte valenza estetica 
e simbolica, che sarà progressivamente superata negli anni successivi, a partire dalla seconda me- tà del XVII secolo, da una precisa e più scarna simbologia codificata.

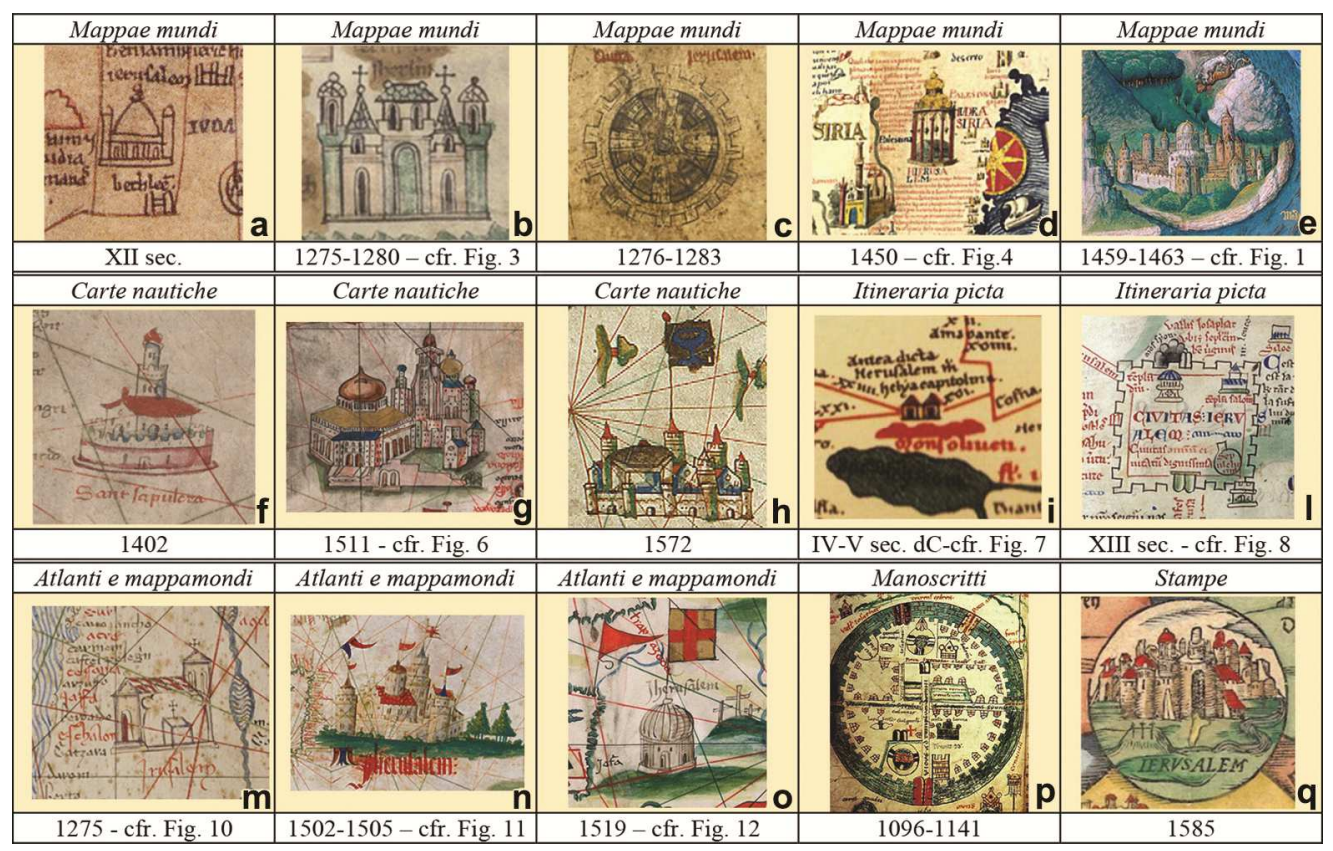

Fig. 13. a) Sawley, Imago Mundi fine XII secolo (ms. 066, p. 2, Corpus Christi College, Cambridge); b) Mappamondo, Saint-Denis cfr. Fig. 3; c) R. di Haldingham, Mappa mundi di Hereford, 1276-1283; d) Fra Mauro, Mappa mundi cfr. Fig. 4; e) La Terra divisa tra i figli di Noè, cfr. Fig 1; f) P. Roselli, Carte marine de l'océan Atlantique Nord-Est de la mer Méditerranée et de la mer Noire, 1402 (CPL GE C-5090 RES, BNF); g) S. de Pilistrina, Carta nautica del Mediterraneo, cfr. Fig. 6; h) J. Holives, Carte de la Méditerranée, 1572 (Cartes nautiques ms. 0488, f. 003, Bibliothèque municipale de Valenciennes); i) Tabula Peutingeriana, cfr. Fig. 7; l) M. Paris, Iter de Londinio in Terram Sanctam, cfr. Fig. 8; m) A. Cresques, Atlante Catalano, cfr. Fig. 10; n) Carta del Cantino, cfr. Fig. 11; o) J. Reinel, Carta del Mondo, cfr. Fig. 12; p) Mappa di Gerusalemme, Gesta Francorum Iherusalem expugnantium, XIII secolo (ms. 776, f. 50v, Bibliothèque d'agglomération de Saint-Omer); q) H. Bünting (1545-1606), Itinerarium Sacrae Scripturae, 1581 (BNF).

\section{Bibliography}

Brotton, J. (2018). La storia del mondo in dodici mappe, Feltrinelli, Milano.

Cantile, A. (2013). Lineamenti di storia della cartografia italiana, Geoweb, Roma.

Conti, S. (2015). "Il lungo cammino della Cartografia", in Pra, E. Del. ed., La storia della cartografia e Martino Martini, Franco Angeli, Milano, pp. 25-46.

Haguet, L. (2018). "L'atlas nautique du Havre, une archéologie documentaire”, in Cahiers havrais de recherche historique, Hors-série, pp. 53-90.

Sansone, S. (2010). "Tra immagine e racconto", in Quintavalle, A. C. Medioevo: le officine, Atti del XII Convegno Internazionale di Studi, I Convegni di Parma, Mondadori Electa Ed., Milano, pp. 434-439.

Umek, D. (2018). "De peregrinatione a Compostela nella cartografia europea tardomedievale (secoli X-XV)", AGEI - Geotema, 58, pp. 142-150. 